\title{
Statistical Learning of Target Location Probability in Children and Adults
}

\author{
Saebyul Lee1, Injae Hong2, and Su Keun Jeong1 \\ 1Cognitive Science Research Group, Korea Brain Research Institute \\ 2Department of Psychology, Yonsei University
}

Corresponding Author: Su Keun Jeong

Address: Korea Brain Research Institute, Cheomdan-ro 61, Dong-gu, Daegu, South Korea, 41062

Email: sukeunjeong@gmail.com

Phone: 82-53-980-8120

\section{Declaration of Conflicting Interests}

The authors declare no conflicts of interest with respect to the authorship or the publication of this article.

\section{Funding}

This work was supported by Korea Brain Research Institute (20-BR-01-10 \& IBS-R001-D12020-b01) funded by the Ministry of Science and ICT. 


\begin{abstract}
Learning environmental regularities allows us to make predictions and guide behavior. Growing evidence of location probability learning (LPL) has shown that the statistical regularity of target locations affects spatial attention allocation. However, past studies on LPL have mostly focused on adults' learning. To achieve a comprehensive understanding of the mechanism of this learning, we investigated the effect of target location probability on 5- to 9-year-old children's visual search in comparison with that of adults. Both children and adults responded faster when the target appeared in the high probability "rich" quadrant than in the low probability "sparse" quadrants of the search space. This attentional bias toward the rich quadrant persisted even when the target was equally likely to appear in all four quadrants. Importantly, the magnitude of the bias was constant across various ages of participants and did not depend on individual differences in executive functions. Taken together, these results provide novel and converging evidence that implicit statistical learning of target locations occurs early in development and remains stable until early adulthood, which is a distinct developmental pattern from explicit goal-driven spatial attention learning.
\end{abstract}

Keywords: location probability learning, probability cuing, implicit learning, development, statistical learning, visual search 


\section{Introduction}

The allocation of attention in space is guided not only by stimulus salience and task demands but also by an observer's past experience. Locations that have frequently contained a visual search target in the past are prioritized in attentional allocation as well as search behavior, which is called "location probability learning” (LPL) (Geng \& Behrmann, 2002; Jiang, Swallow, \& Rosenbaum, 2013; for a review, see Jiang, 2018). Recent studies have shown that this learned spatial bias in visual search is unimpaired even in older adults and patients with Parkinson's disease (Sisk, Twedell, Koutstaal, Cooper, \& Jiang, 2018; Twedell, Koutstaal, \& Jiang, 2017); thus, it was suggested as a compensating attention process for other attentional deficits induced by aging and neurocognitive disease. A complete understanding of LPL should include an understanding of its developmental origins. However, to our knowledge, only two studies have tested LPL in younger participants, which were used only as a control to evaluate autistic children's visual search performance (Jiang, Capistrano, Esler, \& Swallow, 2013; Pellicano et al., 2011). In the current study, we examined when children show adult-like LPL and how it is related to the children's age and their domain-general cognitive development.

LPL has been demonstrated in the visual search paradigm, where the probability of a target appearance was higher in a specific quadrant of the searching space (i.e., the "rich" quadrant). When participants were asked to search for a target (e.g., left-right-tilted T) among distractors (e.g., rotated Ls), they gradually responded faster to the target presented in the rich quadrant. Facilitation in reaction times for the rich quadrant was also observed in the subsequent unbiased phase where a target appeared in all quadrants with equal probability (Jiang et al., 2013) and persisted for at least a week after learning (Jiang, Swallow, Rosenbaum, \& Herzig, 
2013), suggesting that this spatial bias is more likely to be a result of attentional learning than short-term inter-trial facilitation.

A key characteristic of LPL that has been suggested is that this attention bias would be acquired without the intention or explicit awareness of learners. That is, most participants in LPL studies did not notice the biased spatial distribution of target stimuli (Geng \& Behrmann, 2002; Jiang et al., 2013). In addition, few participants who correctly identified the rich quadrant did not show greater LPL than those who did not recognize the uneven target distribution (Twedell et al., 2017). Although a recent meta-analysis has questioned the implicit nature of LPL and suggested a relation between awareness and LPL (Vadillo, Linssen, Orgaz, Parsons, \& Shanks, 2020), LPL still contains considerable features of implicit learning in general.

Generally, implicit learning has been hypothesized to have five distinguishing features from explicit learning (Reber, 1989): (1) developmental invariance (Drag \& Bieliauskas, 2010; Finn et al., 2016), (2) robustness to disease or injury (Reber, 2013; Reber, Martinex, \& Weintraub, 2003), (3) specificity of transfer (Manza \& Reber, 1997), (4) IQ independence (Atwell, Conners, \& Merrill, 2003; Bussy, Charrin, Brun, Curie, \& des Portes, 2011), and (5) secondary task independence (Curran \& Keele, 1993; Hayes \& Broadbent, 1988). That is, implicitly acquired knowledge tends to be inflexible, perceptually bound to the training context, and remains intact with regard to aging, developmental maturation, neurological/psychological disorder, and lack of attentional resources. Likewise, LPL has been shown to be persistent and task specific (Jiang, Swallow, Won, Cistera, \& Rosenbaum, 2015; Salovich, Remington, \& Jiang, 2018), and robust to left hemifield neglects (Geng \& Behrmann, 2002), autistic spectrum disorder (Jiang et al., 2013), aging (Twedell et al., 2017), and Parkinson's disease (Sisk et al., 
2018), as well as secondary task interference (Won \& Jiang, 2015). However, the developmental trajectory of LPL has not been examined empirically.

As was mentioned above, two studies have reported LPL in children aged 5-13 years (Jiang et al., 2013; Pellicano et al., 2011). However, the focal research interest was not in understanding the development of LPL itself but in demonstrating spared LPL in autistic children. Thus, these studies neither included a sufficient number of children with a proper age range to detect age-related changes nor compared their learning to adults'. It is still unknown whether there are any developmental changes in LPL, and when and how children acquire adultlike experience-driven attention learning.

Similar questions have been raised and discussed in the contextual cueing paradigm. Visual search is improved when target-distractor configurations are repeated (Chun \& Jiang, 1998; Sisk, Remington, \& Jiang, 2019). Although the participants learn the target-context association instead of a general spatial bias in the contextual cueing, its learning shows similar characteristics to LPL. For example, both types of learning are mostly implicit and the learning effect persisted even one-week after learning (Chun \& Jiang, 2003; Jiang, Swallow, Rosenbaum, \& Herzig, 2013). Also, the transfer of learning was specific to item color or task difficulty during learning (Jiang \& Song, 2005a) as well as task similarity between learning and testing (Jiang \& Song, 2005b). This contextual cueing effect has been consistently observed in various conditions, but developmental studies with school-aged children have reported conflicting findings. For example, Vaidya, Huger, Howard, and Howard (2007) did not find the cueing effect with 10 year-old-children using the standard T-and-L paradigm. In contrast, Dixon, Zelazo, and De Rosa (2010) reported contextual cueing in children aged 5-9 years using ageappropriate stimuli and a touching response device. These results indicate that with age- 
appropriate stimuli and experimental setting, young children could learn and use spatial context. Meanwhile, using the standard T-and-L task, Couperus, Hunt, Nelson, and Thomas (2011) demonstrated that 10-year-old children's contextual learning was modulated by the ratio between attended and unattended stimuli, in contrast to the robust contextual cueing effect of the adult participants irrespective of attention setting. Additionally, children aged 6-8 years failed to show an adult-like contextual cueing effect when the ratio between the repeated and non-repeated display was low (Yang \& Merrill, 2015). These results suggest that although the basic contextual learning mechanisms may be available early in development, development-dependent learning factors such as available resources of selective attention and working memory may play an important role in the expression of young children's implicit learning.

Given the similarity between LPL and contextual cueing, we may expect to see a similar early developmental onset of basic spatial learning mechanisms with less robustness of the system. However, LPL is distinguished from contextual cueing, in which participants acquire a general spatial attention bias based on simple frequency of target location, but not a higher order target-distractor configuration association that is necessary for predictive use of context. It should be considered that object location is spontaneously encoded and remembered even when it is not task relevant (Foster, Bsales, Jaffe, \& Awh, 2017; Schneegans \& Bays, 2017), but effective encoding of spatial displays has been suggested to require selective attention. Indeed, under reduced attention, when the similarity between target and distractors increased, adult participants' contextual learning was attenuated (Jiang \& Chun, 2001). However, LPL was not affected under reduced attention (Won \& Jiang, 2015). Thus, we presume that such a difference in the underlying learning mechanism between LPL and contextual cueing may lead to a different developmental trajectory. 
In the current study, we first investigated whether LPL appears early in childhood and is maintained at a constant level across development as predicted by the evolutionary model of implicit learning (Reber, 1989), or whether it gradually develops as children's underlying neural and cognitive architectures mature as predicted by the parallel developmental change model between implicit and explicit learning (Thomas et al., 2004). Hence, we included children from various ages between 5 to 9 to increase sensitivity to the detection of developmental differences and compared their performances to the adults'. In addition, we evaluated whether children's LPL would be influenced by development-dependent learning factors, such as executive function and memory.

\section{Methods}

\subsection{Participants}

A total of 49 typically developing children ages 5.04-9.21 years (34 boys, 29 girls; $M$ age $=6.99$ years, $S D=1.15$ years $)$ and 22 healthy adults $(14$ men, 8 women; $M$ age $=22.14$ years, $S D$

$=2.17$ years) participated in the experiment. We split the children into two age groups: 5-6-yearolds (i.e., preschoolers, $N 5-6=26$ ) and 7-9-year-olds (i.e., school-aged, $N 7-9=23$ ). An additional fourteen children participated but were excluded from analysis because of failure to complete the experiment. Based on medium effect size $(f=.25)$, an alpha level of 0.05 and power of 0.95 for repeated measures ANOVA with $2 \times 3$ within-between interactions, a total sample size of $\mathrm{N}=66$ was calculated to be necessary using $\mathrm{G}^{*}$ Power 3.1(Faul, Erdfelder, Lang, \& Buchner, 2007). A medium effect size (.25) was chosen because previous contextual cueing studies that had similar task structures either reported no age-related differences between adults and children or a very small effect size of $\eta_{\mathrm{p}}^{2}=.089$ (Yang \& Merrill, 2015). 
Both adults and children were recruited through advertisements in the neighborhood of Daegu. Adult participants and the parents of child participants provided written consent before the experiment and the children provided verbal assent. All participants had a normal or corrected-to-normal visual acuity and normal color vision, and they were compensated \$10-15 for their participation. All experiments in the current study were approved by the Institutional Review Board of Korea Brain Research Institute.

\subsection{Stimuli and Apparatus}

The experiment was conducted on a 21.5-inch touch monitor with resolution of $1920 \times$ 1080. Participants sat in front of the monitor at their own comfortable distance. MATLAB 2018a with Psychophysics Toolbox (Brainard, 1997) was used to program the experiment. All stimuli were presented on a black background (RGB: 0,0,0). The search items were three different cartoon characters with a single-hand-up position or a hands-down position. The characters with a hands-down position were the targets and the characters with a hand-up position were the distractors. Each display contained a single type of character with one target and seven distractors. Each stimulus subtended 110 px $\times 110$ px. (We report the item size in pixels, not the visual angle, because the distance from the monitor to the participants was not strictly controlled.) The display was divided into a $6 \times 6$ invisible matrix, and each cell was sized $130 \mathrm{px}$ $\times 130 \mathrm{px}$. A white cross with a size of $20 \mathrm{px} \times 20 \mathrm{px}$ was used as a fixation point. Nine cells were allocated to the quadrants. Two cells were randomly selected from each quadrant as item locations. Each item was positioned at the center of the selected cell with a random horizontal or vertical jitter within $\pm 10 \mathrm{px}$. On the search screen, a progress bar to show accumulated correct responses during each session was presented as shown in Figure 1b. The progress bar consisted of a character's small head at the left side (size: $80 \mathrm{px} \times 80 \mathrm{px}$ ) and a white rectangle frame 
$(1730 \mathrm{px} \times 60 \mathrm{px})$. The frame was filled with a yellow square as the participants correctly found the target. The center of the progress bar was 50 px apart from the top edge.

\section{Figure 1}

Schematic description of the experimental design

a)

Training Phase

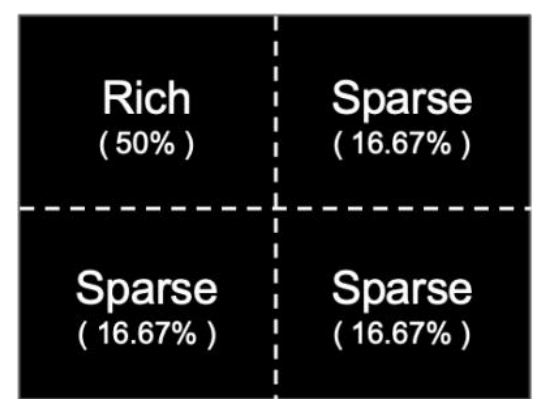

b)

Testing Phase

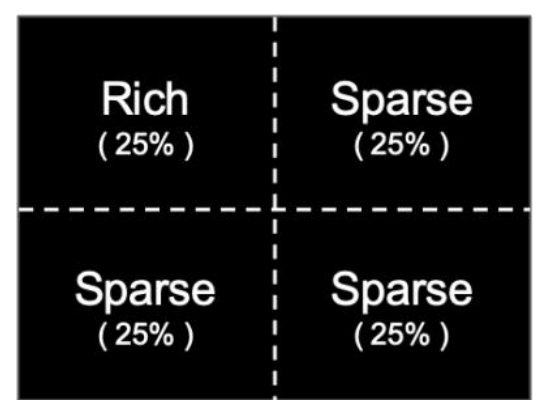

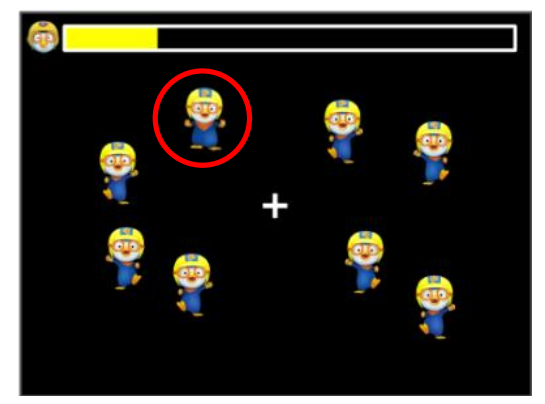

Note. a) The statistical distribution of the training and the testing phase. The display was divided into invisible quadrants. The rich quadrant location was counterbalanced across the participants.

b) Search display example. The character with a hands-down position was a target in this example (the red circle on the target was not shown in the experiment). The items are not drawn to scale.

\subsection{Procedure}

Twenty-four practice trials were given before the main experiment. The main experiment consisted of three sessions to see the time-course change in the magnitude of LPL. Each session contained six blocks, in which the first five blocks were the training phase and the last block was the testing phase. Each block had 24 trials. Each trial started with a fixation cross for $500 \mathrm{ms,}$ then eight search items appeared. The participants searched for a target (hands-down character) 
among distractors (single-hand-up characters) and responded by touching the target. They were instructed to respond as quickly and accurately as possible. Feedback was provided with a smiling face for a correct response for $300 \mathrm{~ms}$ and with a frowning face for an incorrect response for $400 \mathrm{~ms}$. After the feedback, the progress bar showing accumulated correct responses was presented for $500 \mathrm{~ms}$ on the blank screen. The length of the inter-trial interval was 1,000 ms.

Each session started with presenting a new character comprising the target and distractors during the session. The order of the three characters were counterbalanced across participants. During the training phase, unbeknownst to the participants, a target was presented at a designated rich quadrant in $50 \%$ of trials (see Figure 1a). In the remaining $50 \%$ of trials, the target was evenly distributed to three other quadrants with equal probability (16.67\% each). The location of the rich quadrant was counterbalanced across participants but was kept constant across sessions within participants. During the testing phase, every quadrant contained a target with an equal probability $(25 \%)$. The purpose of the testing phase was to test whether the spatial bias in the training phase represented long-term statistical knowledge or short-term inter-trial priming. To keep consistency, although the target was evenly distributed in the searching space during the testing phase, the quadrant was named following the location of the rich/sparse quadrant in the training phase.

At the end of the search task, we asked three questions to verify the participants' awareness of location probability of targets. First, the participants were asked whether they have noticed any rule during the experiment. Regardless of their answer, they were required to state the rule that they conjectured and then to guess the quadrant where the target frequently appeared. The answers were recorded by an experimenter. The participants who successfully 
noticed unequal probability distribution of targets across quadrants or who correctly indicated the rich quadrant in the last question were excluded from final analyses.

Following the search task, to measure children's domain general executive function and memory, the children were tested in four additional tasks - List Sorting Working Memory (LSWM), Picture Sequence Memory (PSM), Flanker Task, and Dimensional Change Card Sorting (DCCS) - among the NIH toolbox cognitive battery (Weintraub et al., 2013). The NIH toolbox cognitive battery was administered on a 10.5-inch iPad Pro. The order of tasks was pseudo-randomized across participants. Although the tasks were computerized, an experimenter presented task instructions orally to the children and monitored their performance. Corrected standard scores with a standardized mean of $100(S D=15)$ were used for the analyses.

$\boldsymbol{L S W M}$. In this task, the children were shown a list of stimuli both visually and auditorily on the iPad, and then they were asked to repeat all of the stimuli back to the experimenter in order of increasing real-world size, from smallest to largest. The children were first shown a list with two items; if they succeeded on this two-item list, the length of the list increased by one item, up to a total seven-item list. If the children failed on a given trial, they received another trial with the same length of list, and if they failed on that trial again, the test was terminated. The number of stimuli in the final list that the children successfully recalled provides a measure of working memory capacity.

PSM. This task measures children's episodic memory retrieval. Color-illustrated sequences of pictures were shown to the children in the center of the iPad screen in a fixed order. When each picture appeared, the experimenter briefly described it. Once all the pictures of the sequence were displayed the pictures were randomly distributed at the center of the screen. The children were instructed to locate all the pictures in their proper position of the sequence to 
reproduce the correct order. The length of the sequence that the children were asked to order was determined by their age.

Flanker Task. This task was included to measure children's inhibitory control in the context of selective visual attention. Participants were required to indicate the left-right orientation of a centrally presented stimulus with corresponding button pressing on the screen, while inhibiting surrounding stimuli (i.e., flankers) that had either congruent or incongruent orientation to the central stimulus. The task contains an easier version (fish stimuli) and a more difficult version (arrow stimuli) depending on the children's age. Changes in response time from the congruent trials to the incongruent trials provides a measure of inhibitory control.

DCCS. This task is a measure of cognitive flexibility, also known as task switching or set shifting. The children were instructed to match a centrally presented test stimulus to one of two lateralized target stimuli either by shape or by color with touching the matching target stimulus. After sorting test stimuli according to one dimension either by color or shape (pre-switch block), which was counterbalanced, sorting dimension was switched (switch block). If the children succeeded in both the pre-switch and switch blocks, the mixed block consisting of both color and shape matching tasks was provided. DCCS scores were calculated based on both accuracy and reaction time.

\section{Results}

One adult participant was excluded from analysis because the participant had explicit knowledge of the probability manipulation as well as the rich quadrant location. Fourteen children failed to complete the task. We first analyzed the data from 49 children who completed all three sessions of the experiment and then ran additional analyses with all participants regardless of completion of the experiment. Trials with reaction times (RTs) beyond 10 seconds 
were excluded (Jiang et al., 2014; Won \& Jiang, 2015) resulting in an elimination $1.97 \%$ of trials from children and none from adults. The children's accuracy was 98.44\% $(S D=1.49 \%)$, while the adults' accuracy was $98.33 \%(S D=1.28 \%)$. RTs in the correct trials were analyzed. The RT data from three sessions were collapsed, because all sessions had an identical structure with the same location probability except for the character type. The first five blocks in each session belonged to the training phase and the last one belonged to the testing phase. All statistical analyses were conducted in JASP 0.12.2 (JASP Team, 2020).

\subsection{The Acquisition of LPL in the Training Phase}

We first investigated the effect of age group on the acquisition of LPL using a mixed factor ANOVA on the participants' RTs during the training phase. The typical LPL would be presented as the facilitation of target detection when the target appears at a rich quadrant compared to the sparse quadrants. The ANOVA model included probability (rich, sparse) and session (1-3) as within-subject factors and age group (5-6-year-olds, 7-9-year-olds, adults) as a between-subjects factor. Simple main effects of probability, $F(1,68)=131.290, p<.001, \eta_{p^{2}}$ $=.659$, and age group, $F(2,68)=88.632, p<.001, \eta_{\mathrm{p}}^{2}=.723$, were significant. That is, the RTs in the rich condition were significantly faster than the RTs in the sparse condition, indicating the acquisition of a spatial bias toward the frequent target location (see Figure 2a). The age group effect showed a linear trend, $\beta=-1.540, t(68)=-13.290, p<.001$, indicating that overall $\mathrm{RTs}$ decreased as the participants' age increased. Meanwhile, the main effect of session was not significant, $F(2,136)=2.929, p=.057, \eta_{\mathrm{p}}{ }^{2}=.041$. The interaction effect between probability and session was significant with the Greenhouse-Geisser correction, $F(1.579,107.360)=17.258$, $p<.001, \eta_{\mathrm{p}}^{2}=.202$, indicating that the extent of acquired spatial bias significantly changed over the sessions. However, any other interaction effect related to age groups was not significant: 
probability $\times$ age interaction, $F(2,68)=2.386, p=.100, \eta_{\mathrm{p}}^{2}=.066$; session $\times$ age interaction, $F(4,136)=1.801, p=.132, \eta_{\mathrm{p}}^{2}=.050 ;$ probability $\times$ age $\times$ session interaction, $F(3.158,107.360)$ $=.656, p=.588, \eta_{\mathrm{p}}^{2}=.019$. That is, neither the acquisition of spatial bias nor the pace of bias acquisition differed across the age groups.

\section{Figure 2}

The results of the experiment

a)

Learning

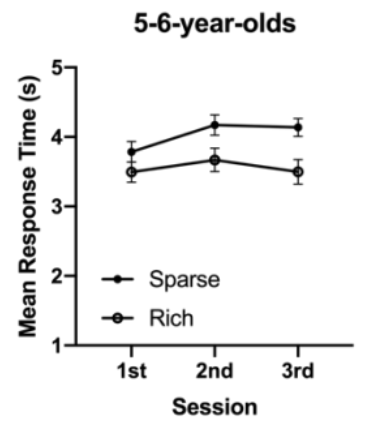

b)

Testing

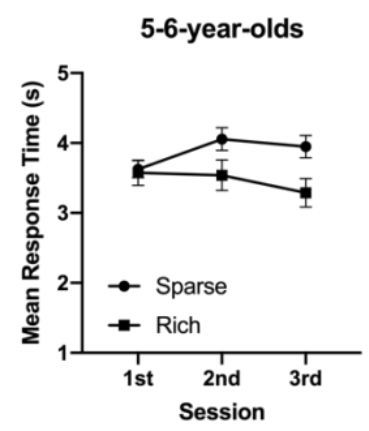

7-9-year-olds

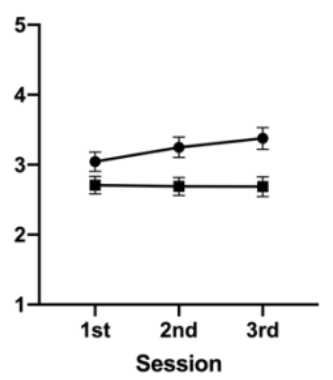

7-9-year-olds

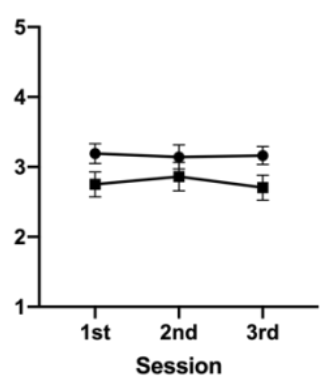

Adults

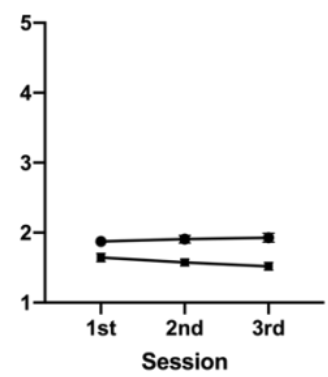

Adults

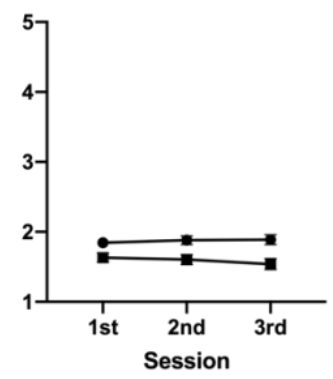

Note. a) RTs in the learning phase by sessions and by age groups. The error bars indicate

standard error of mean. b) RTs in the testing phase by sessions and by age groups. The error bars indicate standard error of mean. 
To assess the evidence for and against the presence of the age-related interaction effect, we submitted the RTs to a Bayesian mixed factor ANOVA with probability condition, session number, and age groups. Examination of the Q-Q plots suggested that the assumption of normality was not violated. The model including main effects (probability, session, and age group) and a probability $\times$ session interaction was most supported by the data, $B F_{10}=$ $2.406 \times 10^{44}$, whereas adding the probability by age interaction decreased the degree of this support by a factor of $1.0 / 0.662=1.51$. These results indicate that the data provided decisive evidence for the acquisition of LPL early in development, but the evidence against age-related differences in LPL was inconsequential.

\subsection{The Persistence of LPL in the Testing Phase}

In the testing phase, contrary to the training phase, a target appeared in all four quadrants with equal probability. Accordingly, the spatial bias in the testing phase cannot be explained by short term inter-trial response priming. Instead, spatial bias toward the rich quadrant would indicate that the participants successfully acquired long-term statistical knowledge. A mixed factor ANOVA with probability (rich, sparse) and session (1-3) as within-subject factors and age group (5-6-year-olds, 7-9-year-olds, adults) as a between-subjects factor was conducted to explore the existence of long-term statistical knowledge across different age groups (Figure 2b). The main effects of probability and age group were significant, $F(1,68)=37.607, p<.001, \eta_{\mathrm{p}}^{2}$ $=.356, \mathrm{~F}(2,68)=82.497, \mathrm{p}<.001, \eta_{\mathrm{p}}^{2}=.708$, respectively. These results implied that the LPL persisted even after the probability of target distribution was equal across all quadrants, but the overall RTs differed by age group. As in the training, the main effect of session was not significant, $F(2,136)=.780, p=.461, \eta_{\mathrm{p}}{ }^{2}=.011$, but the interaction effect between probability and session was significant, $F(2,136)=3.087, p=.049, \eta_{\mathrm{p}}^{2}=.043$. Any other interaction effect 
related to age groups was not significant: probability $\times$ age interaction, $F(2,68)=.470, p=.627$, $\eta_{\mathrm{p}}{ }^{2}=.014 ;$ session $\times$ age interaction, $F(4,136)=.331, p=.856, \eta_{\mathrm{p}}{ }^{2}=.010 ;$ probability $\times$ age $\times$ session interaction, $F(4,107.360)=.656, p=.588, \eta_{\mathrm{p}}^{2}=.019$. The results from the testing phase demonstrated that controlling for intertrial repetitions, the learned spatial bias still preserved regardless of the participant's age, and the bias that learned during searching for a specific item could be transferred to searching for a different item.

A Bayesian mixed factor ANOVA resulted in a probability and age main effects only model as the best-representing model of the data compared to the null model, $B F_{10}=$ $4.078 \times 10^{23}$, indicating decisive evidence in favor of the persistence of LPL and age-related decrease in overall RTs. The addition of probability $\times$ age group interaction decreased the extent of this support by a factor of $1 / .076=13.157$. Adding the main effect of session order with the probability $\times$ age group interaction dropped the BF by $1 / .023=43.478$ factor when compared to the best model. These results showed positive evidence for developmental invariance in the persistence of LPL. Thus, the results of the Bayesian ANOVAs from both the training and testing phases exhibited decisive evidence of the robust acquisition and persistence of LPL across different age groups.

To control for the different baseline RTs across participants, we generated a standardized cueing index. The standardized cueing index could be a better measure than the raw RT difference between the sparse and rich conditions because it takes into account overall RT differences when determining the magnitude of LPL. The standardized cueing index was calculated based on averaged RTs in each block with the following equation:

$$
\text { Cueing index }=\frac{(\text { RTs of sparse condition }-R T s \text { of rich condition })}{(R T s \text { of sparse condition }+R T \text { of rich condition })}
$$


A zero value of the cueing index indicates the absence of spatial bias either toward the rich or sparse target locations. A larger cueing index indicates a greater spatial bias toward the frequent target location.

A mixed factor ANOVA was applied to cueing indices in the testing phase with the order of session (1-3) as a within-subject factor and age group as a between-subjects factor. The effect of the session order was significant, $F(1.763,119.880)=21.266, p<.001, \eta_{\mathrm{p}}{ }^{2}=.238$, with its linear trend, $\beta=.038, t(136)=6.494, p<.001$. Other effects and interaction were not significant. This result indicates that the extent of the spatial bias increased as sessions progressed regardless of the target item identity, however the magnitude and increasing trend of the bias did not differ across different age groups, $F(2,68)=1.472, p=.237, \eta_{\mathrm{p}}{ }^{2}=.026 ; F(3.526,119.880)=21.266, p$ $<.001, \eta_{\mathrm{p}}^{2}=.238$, respectively.

As was done in the analyses of raw RT difference above, we assessed the evidence for and against the presence of the age-related effect on the magnitude of LPL using a Bayesian mixed factor ANOVA with the order of session and age groups as factors. The session order main effect only model was best supported by the data compared to the null model, $B F_{10}=$ $2.132 \times 10^{6}$, which indicates decisive evidence for an increase in the magnitude of LPL as learning progressed. However, the addition of either age main effect or session $\times$ age group interaction dropped the degree of this support by a factor of $1 / .434=2.304$ and $1 / .014=72.428$, respectively. Although the results showed strong evidence against age-related differences in the learning effect of LPL, the evidence against age-related difference in the magnitude of LPL was inconsequential $\left(B F_{10}=2.304\right)$. Thus, we considered the inclusion Bayes factor for each of the model's predictor based on all possible models simultaneously and observed decisive evidence for the inclusion of the order of session, $B F_{\text {incl }}=1.513 \times 10^{6}$, but neither for the inclusion of 
age group, $B F_{\text {incl }}=.314$, nor for the inclusion of the session order $\times$ age group interaction, $B F_{\text {incl }}=.041$

The overall results demonstrated that the children and adults gradually acquired a spatial bias toward the rich quadrant, and they accumulated their learning experiences regardless of identity change in target items between sessions. This learned spatial bias was preserved even when the targets were evenly distributed in the searching space, and its magnitude was constant across age groups. These results implied that the acquisition and persistence of LPL could be developmentally invariant, unlike explicit attention learning.

\subsection{The Effect of Development-dependent Factors on the Children's LPL}

Even though the group analyses of LPL resulted in insignificant age-related effects, there was substantial variability in children's LPL, indicating that there may have been individual differences in their LPL. Hence, we evaluated whether development-dependent learning factors (on top of chronological age) such as executive function and memory would affect children's LPL. It has been known that the form of difference scores (i.e., differences in response time between conditions, such as congruent and incongruent conditions of a Stroop task) could increase measurement errors and decrease reliability (Cohen \& Cohen, 1983; Friedman \& Miyake, 2004; Hedge, Powel, \& Sumner, 2018), resulting in low correlations between the difference scores and other individual ability scores. Therefore, instead of using the cueing index as the measure of LPL, we used the residual of RTs in the rich condition after regressing out RTs in the sparse condition, reasoning that the residual should be a combination of probability cueing effect and measurement error (Cronbach \& Furby, 1970; Friedman \& Miyake, 2004).

RTs in the sparse condition predicted $55.2 \%$ of the variance in RTs in the rich condition. The residual variance $(54 \%)$ was significantly greater than zero, $t(48)=3.789, p<.001$, Cohen's 
$d=.541$, suggesting RTs in the rich condition contained enough variance not attributable to baseline response latency. The reliability estimate of the residualized difference scores was reasonable, Cronbach's $\alpha=.616,95 \%$ confidence interval $=[0.320,0.780]$. Descriptive statistics for NIH toolbox measures, the residualized difference score of LPL, and their correlations are presented in Table 1. Since the toolbox tasks were given after performing the search task, some children refused to participate in more than two toolbox tasks because of fatigue. Among 49 children who completed all sessions of the search task, 29 children also finished all toolbox tasks, but 20 children did not complete more than one tool box task.

Table 1.

Descriptive Statistics and Correlations for the Variables of Interest

\begin{tabular}{|c|c|c|c|c|c|c|c|c|c|}
\hline & $\begin{array}{l}\text { Mean } \\
\text { (SD) }\end{array}$ & 1 & 2 & 3 & 4 & 5 & & 6 & 7 \\
\hline 1. PSM (long-term memory) & $\begin{array}{l}99.161 \\
(22.18)\end{array}$ & - & & & & & & & \\
\hline 2. LSWM (working memory) & $\begin{array}{c}89.102 \\
(19.467)\end{array}$ & .099 & - & & & & & & \\
\hline 3. DCCS (set switching) & $\begin{array}{l}84.094 \\
(27.33)\end{array}$ & .145 & $.463 *$ & - & & & & & \\
\hline 4. Flanker (inhibitory control) & $\begin{array}{c}93.082 \\
(19.727)\end{array}$ & .047 & $.716 * * *$ & .078 & - & & & & \\
\hline 5. Cueing Index (LPL) & $\begin{array}{l}.068 \\
(.086)\end{array}$ & .094 & .002 & -.304 & .145 & - & & & \\
\hline $\begin{array}{l}\text { 6. Residualized Difference of } \\
\text { LPL }\end{array}$ & $\begin{array}{l}.008 \\
(.556)\end{array}$ & -.109 & .015 & .317 & $-.095 * *$ & -.973 & $* * *$ & - & - \\
\hline 7. Age & $\begin{array}{c}6.998 \\
(1.153)\end{array}$ & .031 & $.309 *$ & .254 & $.364 *$ & .034 & & .058 & - \\
\hline
\end{tabular}

Note: PSM, Picture Sequence Memory; LSWM, List Sorting Working Memory; DCCS, Dimensional Change Card Sorting Test; LPL, location probability learning $* p<.05, * * p<.01, * * * p<.001$ 
We first verified that there were age-related changes in children's executive function by regressing each task scores on their age. Children's age was a significant predictor for their working memory $(\mathrm{LSWM}), \beta=5.220, F(1,47)=4.842, p=.033$, and inhibitory control (Flanker task), $\beta=6.226, F(1,47)=7.024, p=.011$, but not for cognitive flexibility (DCCS) scores, $\beta=$ $3.460, F(1,30)=2.000, p=.168$, and episodic memory retrieval $(\mathrm{PSM}), \beta=.549, F(1,31)=$ $0.029, p=.866$. Children's age predicted neither the change in the cueing index nor that in the residualized difference score as shown in Figure $3: \beta=.003, F(1,47)=.055, p=.816 ; \beta=-.028$, $F(1,47)=.157, p=.693$, respectively

\section{Figure 3}

The nonsignificant age effect on the magnitude of children's LPL

a)

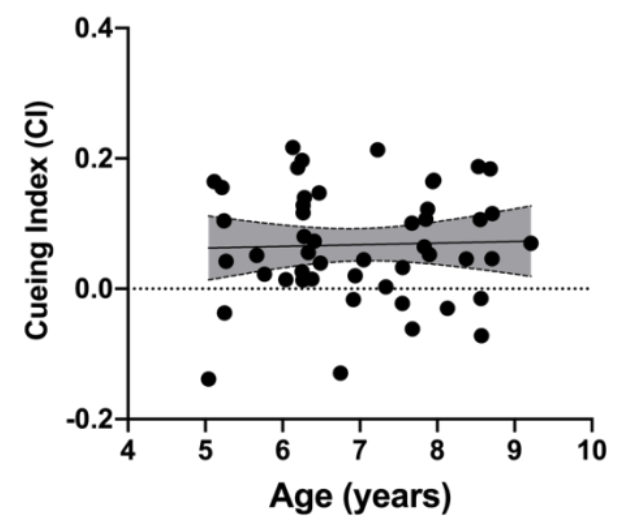

b)

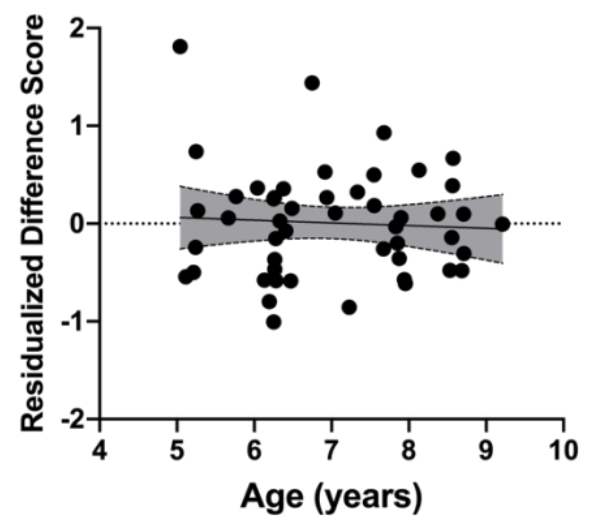

Note. a) Regression line between children's age and cueing index in the testing phase. b) Regression line between children's age and residualized difference score in the testing phase. Individual circles match to the individual data points. Shaded area reflects $95 \%$ confidence interval. 
Then, we carried out a Bayesian multiple regression analysis for the children's residualized difference scores in the testing phase with standardized scores of LSWM, PSM, Flanker, and DCCS tests, and their age as predictors, so as to address whether these individual differences in executive function affected children's LPL. An uninformed uniform prior, $\mathrm{P}(\mathrm{M})$ $=.031$ was set for each of all possible models. However, no possible regression model resulted in considerable evidence against the null model, as shown in Table 2 (i.e., Residualized Difference Models). The $95 \%$ of credible intervals of all the covariates included in the analysis contained zero inside: Age, [-0.067, 0.089]; PSM, [-0.007, 0.002]; LSWM, [-4.284× $\left.10^{-4}, 0.017\right]$; DCCS, $[-0.002,0.013]$; flanker, $[-0.011,0.004]$. These results suggest that the magnitude of LPL was not only developmentally invariant but also independent of individual differences in executive function and memory.

To account for the possibility that the search task itself might not be sensitive enough to catch age-related or individual differences for some reason, we applied similar Bayesian multiple regression models to the overall RTs. The overall RTs model including working memory and children's age provided strong evidence against the null model, $B F_{10}=21.267$, and other models including the set switching and inhibition as well as working memory and age also showed strong evidence against the null model (see Overall RT Models in Table 2).

Thus, although the search task and cueing indices were sensitive enough to detect agerelated or individual differences, the children's long-term LPL during testing was not correlated with their domain-general cognitive development. These results confirmed the notion that LPL is distinguishable from the explicit learning mechanism, in that LPL is developmentally invariant and independent from development-dependent learning factors. 
Table 2.

Subsets of 4 best Bayesian Linear Regression Models for Predicting Variables of Children's Visual Search Performances

\begin{tabular}{|c|c|c|c|c|c|}
\hline Models & $\mathrm{P}(\mathrm{M})$ & $\mathrm{P}(\mathrm{M} \mid$ data $)$ & $\mathrm{BF} \mathrm{M}$ & $\mathrm{BF}_{10}$ & $\mathrm{R}^{2}$ \\
\hline \multicolumn{6}{|l|}{ Residualized Difference Models } \\
\hline Null model & .031 & .047 & 1.543 & 1.000 & .000 \\
\hline DCCS & .031 & .083 & 2.800 & 1.747 & .142 \\
\hline LWSM & .031 & .082 & 2.781 & 1.737 & .141 \\
\hline LWSM + flanker & .031 & .065 & 2.146 & 1.365 & .195 \\
\hline LWSM + DCCS & .031 & .063 & 2.069 & 1.320 & .192 \\
\hline \multicolumn{6}{|l|}{ Overall RT Models } \\
\hline Null model & .031 & .007 & .2173 & 1.000 & .000 \\
\hline LSWM + Age & .031 & .148 & 5.365 & 21.267 & .381 \\
\hline Age & .031 & .135 & 4.854 & 19.517 & .308 \\
\hline $\mathrm{LSWM}+\mathrm{DCCS}+\mathrm{Age}$ & .031 & .097 & 3.348 & 14.050 & .416 \\
\hline LSWM + DCCS + flanker + Age & .031 & .092 & 3.133 & 13.231 & .464 \\
\hline
\end{tabular}

Note: $\mathrm{P}(\mathrm{M})=$ prior model probability; $\mathrm{P}(\mathrm{M} \mid$ data $)=$ posterior model probability; $\mathrm{BF} \mathrm{M}=$ change from prior model odds to posterior model odds; $\mathrm{BF}_{10}=$ Bayes factor for each model against the null model

\section{Discussion}

The aims of the current investigation were 1) to determine when and how children acquire adult-like LPL and 2) to examine whether the acquisition of LPL is affected by development-dependent learning factors. To our knowledge, this is the first empirical investigation of the developmental nature of LPL making direct comparisons to adults' LPL. With age-appropriate stimuli and response methods, children aged 5-9 years successfully learned 
the statistical regularity of the target location and used this knowledge to guide their spatial attention. The extent of their learned spatial bias in the testing phase was also equivalent to adults' learning. Furthermore, the children's LPL was independent of their executive functions.

Our results have several implications for understanding the nature of LPL. First, our data provide evidence that children can learn the probability of target locations and use it to guide their spatial attention without explicit awareness. The magnitude of their learned spatial bias was comparable to adults' learning, suggesting the presence of a highly functioning implicit spatial attention mechanism early in development. This is consistent with findings showing early mature implicit learning (Amso \& Davidow, 2012; Aslin, 2017; Finn et al., 2016; Thomas et al., 2004). In light of previous findings on older adults' spared LPL (Jiang, 2018; Twedell et al., 2017), the current investigation added evidence to demonstrate a developmental trajectory of stable LPL from young childhood to late adulthood.

Second, our results revealed the independence of the children's LPL from domaingeneral cognitive development. While the children's age, working memory capacity, and inhibitory control successfully predicted overall search efficiency, the magnitude of LPL was not related to these factors. Independence of general fluid intelligence has been considered as a significant feature of implicit learning (Dienes \& Berry, 1997) because implicit learning is believed to be dependent on the basal ganglia memory system separately from the prefrontal cortex- and medial temporal lobe-dependent memory system (Janacsek \& Nemeth, 2013; Poldrack et al., 2001). Independence of children's LPL from working memory corresponds to the previous study showing that a secondary working memory task did not interfere with adults' LPL (Won \& Jiang, 2015). Parkinson's patients, who often have impaired working memory and executive function, also showed unimpaired LPL (Sisk et al., 2018). Overall, our results confirm 
that the underlying mechanism serving LPL is qualitatively different from that of explicit learning.

It is well-known that goal-driven attention control undergoes a great deal of developmental change that extends over a prolonged period of development (e.g., Lookadoo, Yang, \& Merrill, 2017; Rueda et al., 2004). Rather, our data demonstrated stable LPL from early childhood to early adulthood, which has distinguishable characteristics from goal-driven attention, such as being implicit, inflexible, resource-independent, and task-specific. LPL has been proposed as a distinctive form of procedural/habitual attention acquired through reinforcement learning (Jiang et al., 2013), compensating for a decline, and protracted development of goal-driven attention. As people may guide their attention habitually rather than declaratively under distraction, time pressure, and limited task ability, young children experiencing more difficulty in suppressing singleton distractors may overcome this interference by deploying habitual attention control when distractors appear in a repeated display (Goschy et al., 2014; Wang, Samara, \& Theeuwes, 2019).

What precisely do children learn from environmental regularity? Does repeated target location guide children's spatial attention to important locations during a search, or does it speed up planning and execution of response after finding a target by inter-trial response repetition? Our data showed that RT facilitation in the rich quadrant remained persistent even when the target location was evenly distributed in the search space. Without response repetition, the children still responded faster to the target in a rich location. Previous adult studies revealed that the first saccadic eye movement (within $200 \mathrm{~ms}$ after stimuli onset) was directed toward the rich region (Jiang, Won, \& Swallow, 2014) and LPL occurred even when the participants were not allowed to move their eyes (Jiang et al., 2014). Similar to adults, it is likely that children in the 
current study learned spatial attention bias instead of response repetition or oculomotor routine. However, it is still possible that children learned a routine of dexterous hand movements instead of spatial attention bias because our search task directly associated finger tapping response with target selection, unlike previous adult studies. Further studies will be necessary to fully dissociate spatial attentional bias from motor learning.

To summarize, these results suggest that location probability learning ability appears early in childhood and is maintained at a constant level across development, as suggested by the developmental invariance approach of implicit learning. These results contribute to making progress toward elucidating the developmental origin of the habitual attention system. 


\section{References}

Amso, D., \& Davidow, J. (2012). The development of implicit learning from infancy to adulthood: Item frequencies, relations, and cognitive flexibility. Developmental Psychobiology, 54(6), 664-673. https://doi.org/10.1002/dev.20587

Aslin, R. N. (2017). Statistical learning: a powerful mechanism that operates by mere exposure. Wiley Interdisciplinary Reviews: Cognitive Science, 8(1-2), 1-7. https://doi.org/10.1002/wcs.1373

Atwell, J. A., Conners, F. A., \& Merrill, E. C. (2003). Implicit and Explicit Learning in Young Adults With Mental Retardation. American Journal on Mental Retardation, 108(1), 56. https://doi.org/10.1352/0895-8017(2003)108<0056:IAELIY>2.0.CO;2

Brainard, D. H. (1997). The Psychophysics Toolbox. Spatial Vision, 10(4), 433-436. https://doi.org/10.1163/156856897X00357

Bussy, G., Charrin, E., Brun, A., Curie, A., \& des Portes, V. (2011). Implicit procedural learning in fragile X and Down syndrome. Journal of Intellectual Disability Research, 55(5), 521528. https://doi.org/10.1111/j.1365-2788.2011.01410.x

Chun, M. M., \& Jiang, Y. (1998). Contextual Cueing: Implicit Learning and Memory of Visual Context Guides Spatial Attention. Cognitive Psychology, 36(1), 28-71. https://doi.org/10.1006/cogp.1998.0681

Cohen, J., \& Cohen, P. (1983). Applied multiple regression/correlation for the behavioral sciences. Hillsdale, NJ: Lawrence Earlbaum.

Couperus, J. W., Hunt, R. H., Nelson, C. A., \& Thomas, K. M. (2011). Visual search and contextual cueing: Differential effects in 10-year-old children and adults. Attention, Perception, and Psychophysics, 73(2), 334-348. https://doi.org/10.3758/s13414-010-0021-6 
Cronbach, L. J., \& Furby, L. (1970). How we should measure "change”: Or should we? Psychological Bulletin, 74(1), 68-80. https://doi.org/10.1037/h0029382

Curran, T., \& Keele, S. W. (1993). Attentional and nonattentional forms of sequence learning. Journal of Experimental Psychology: Learning, Memory, and Cognition, 19(1), 189-202. https://doi.org/10.1037/0278-7393.19.1.189

Dienes, Z., \& Berry, D. (1997). Implicit learning: Below the subjective threshold. Psychonomic Bulletin \& Review, 4(1), 3-23. https://doi.org/10.3758/BF03210769

Dixon, M. L., Zelazo, P. D., \& De Rosa, E. (2010). Evidence for intact memory-guided attention in school-aged children. Developmental Science, 13(1), 161-169. https://doi.org/10.1111/j.1467-7687.2009.00875.x

Drag, L. L., \& Bieliauskas, L. A. (2010). Contemporary Review 2009: Cognitive Aging. Journal of Geriatric Psychiatry and Neurology, 23(2), 75-93. https://doi.org/10.1177/0891988709358590

Faul, F., Erdfelder, E., Lang, A.-G., \& Buchner, A. (2007). G*Power 3: A flexible statistical power analysis program for the social, behavioral, and biomedical sciences. Behavior Research Methods, 39(2), 175-191. http://doi.org/10.3758/BF03193146

Finn, A. S., Kalra, P. B., Goetz, C., Leonard, J. A., Sheridan, M. A., \& Gabrieli, J. D. E. (2016). Developmental dissociation between the maturation of procedural memory and declarative memory. Journal of Experimental Child Psychology, 142, 212-220. https://doi.org/10.1016/j.jecp.2015.09.027

Foster, J. J., Bsales, E. M., Jaffe, R. J., \& Awh, E. (2017). Alpha-Band Activity Reveals Spontaneous Representations of Spatial Position in Visual Working Memory. Current Biology, 27(20), 3216-3223.e6. https://doi.org/10.1016/j.cub.2017.09.031 
Friedman, N. P., \& Miyake, A. (2004). The Relations Among Inhibition and Interference Control Functions: A Latent-Variable Analysis. Journal of Experimental Psychology: General, 133(1), 101-135. https://doi.org/10.1037/0096-3445.133.1.101

Geng, J. J., \& Behrmann, M. (2002). Probability cuing of target location facilitates visual search implicitly in normal participants and patients with hemispatial neglect. Psychological Science, 13(6), 520-525. https://doi.org/10.1111/1467-9280.00491

Goschy, H., Bakos, S., Mueller, H. J., \& Zehetleitner, M. (2014). Probability cueing of distractor locations: Both intertrial facilitation and statistical learning mediate interference reduction. Frontiers in Psychology, 5(OCT), 1-11. https://doi.org/10.3389/fpsyg.2014.01195

Hayes, N. A., \& Broadbent, D. E. (1988). Two modes of learning for interactive tasks. Cognition, 28(3), 249-276. https://doi.org/10.1016/0010-0277(88)90015-7

Hedge, C., Powell, G., \& Sumner, P. (2018). The reliability paradox: Why robust cognitive tasks do not produce reliable individual differences. Behavior Research Methods, 50(3), 11661186. https://doi.org/10.3758/s13428-017-0935-1

Janacsek, K., \& Nemeth, D. (2013). Implicit sequence learning and working memory: Correlated or complicated? Cortex, 49(8), 2001-2006. https://doi.org/10.1016/j.cortex.2013.02.012

JASP Team (2020). JASP (Version 0.12.2) [Computer software].

Jiang, Y. V. (2018). Habitual versus goal-driven attention. Cortex, 102, 107-120. https://doi.org/10.1016/j.cortex.2017.06.018

Jiang, Y. V., Capistrano, C. G., Esler, A. N., \& Swallow, K. M. (2013). Directing attention based on incidental learning in children with autism spectrum disorder. Neuropsychology, 27(2), 161-169. https://doi.org/10.1037/a0031648 
Jiang, Y. V., \& Chun, M. M. (2001). Selective attention modulates implicit learning. The Quarterly Journal of Experimental Psychology Section A, 54(4), 1105-1124. https://doi.org/10.1080/713756001

Jiang, Y. V., Swallow, K. M., \& Rosenbaum, G. M. (2013). Guidance of spatial attention by incidental learning and endogenous cuing. Journal of Experimental Psychology: Human Perception and Performance, 39(1), 285-297. http://doi.org/10.1037/a0028022

Jiang, Y. V., Swallow, K. M., Rosenbaum, G. M., \& Herzig, C. (2013). Rapid acquisition but slow extinction of an attentional bias in space. Journal of Experimental Psychology: Human Perception and Performance, 39(1), 87-99. http://doi.org/10.1037/a0027611

Jiang, Y. V., Swallow, K. M., Won, B.-Y., Cistera, J. D., \& Rosenbaum, G. M. (2015). Task specificity of attention training: the case of probability cuing. Attention, Perception, \& Psychophysics, 77(1), 50-66. https://doi.org/10.3758/s13414-014-0747-7

Jiang, Y. V., Won, B., \& Swallow, K. M. (2014). First saccadic eye movement reveals persistent attentional guidance by implicit learning. Journal of Experimental Psychology: Human Perception and Performance, 40(3), 1161-1173. https://doi.org/10.1037/a0035961

Lookadoo, R., Yang, Y., \& Merrill, E. C. (2017). Encouraging top-down attention in visual search:A developmental perspective. Attention, Perception, and Psychophysics, 79(7), 2007-2020. https://doi.org/10.3758/s13414-017-1379-5

Manza, L., \& Reber, A. S. (1997). Representing artificial grammars: Transfer across stimulus forms and modalities. In How Implicit Is Implicit Learning? (pp. 73-106). Oxford University Press. https://doi.org/10.1093/acprof:oso/9780198523512.003.0004

Pellicano, E., Smith, A. D., Cristino, F., Hood, B. M., Briscoe, J., \& Gilchrist, I. D. (2011). Children with autism are neither systematic nor optimal foragers. Proceedings of the 
National Academy of Sciences of the United States of America, 108(1), 421-426. https://doi.org/10.1073/pnas.1014076108

Poldrack, R. A., Clark, J., Paré-Blagoev, E. J., Shohamy, D., Creso Moyano, J., Myers, C., \& Gluck, M. A. (2001). Interactive memory systems in the human brain. Nature, 414(6863), 546-550. https://doi.org/10.1038/35107080

Reber, A. S. (1989). Implicit Learning and Tacit Knowledge. Journal of Experimental Psychology: General, 118(3), 219-235. https://doi.org/10.1037/0096-3445.118.3.219

Reber, P. J. (2013). The neural basis of implicit learning and memory: a review of neuropsychological and neuroimaging research. Neuropsychologia, 51(10), 2026-2042. https://doi.org/10.1016/j.neuropsychologia.2013.06.019

Reber, P. J., Martinez, L. A., \& Weintraub, S. (2003). Artificial grammar learning in Alzheimer's disease. Cognitive, Affective, \& Behavioral Neuroscience, 3(2), 145-153.

Rueda, M. R., Fan, J., McCandliss, B. D., Halparin, J. D., Gruber, D. B., Lercari, L. P., \& Posner, M. I. (2004). Development of attentional networks in childhood. Neuropsychologia, 42(8), 1029-1040. https://doi.org/10.1016/j.neuropsychologia.2003.12.012

Schneegans, S., \& Bays, P. M. (2017). Neural architecture for feature binding in visual working memory. Journal of Neuroscience, 37(14), 3913-3925. https://doi.org/10.1523/JNEUROSCI.3493-16.2017

Sisk, C. A., Remington, R. W., \& Jiang, Y. V. (2019). Mechanisms of contextual cueing: A tutorial review. Attention, Perception, \& Psychophysics, 81(8), 2571-2589. https://doi.org/10.3758/s13414-019-01832-2

Sisk, C. A., Twedell, E. L., Koutstaal, W., Cooper, S. E., \& Jiang, Y. V. (2018). Implicitlylearned spatial attention is unimpaired in patients with Parkinson's disease. 
Neuropsychologia, 119, 34-44. https://doi.org/10.1016/j.neuropsychologia.2018.07.030

Thomas, K. M., Hunt, R. H., Vizueta, N., Sommer, T., Durston, S., Yang, Y., \& Worden, M. S. (2004). Evidence of developmental differences in implicit sequence learning: An fMRI study of children and adults. Journal of Cognitive Neuroscience, 16(8), 1339-1351. https://doi.org/10.1162/0898929042304688

Twedell, E. L., Koutstaal, W., \& Jiang, Y. V. (2017). Aging affects the balance between goalguided and habitual spatial attention. Psychonomic Bulletin \& Review, 24(4), 1135-1141. https://doi.org/10.3758/s13423-016-1214-3

Vadillo, M. A., Linssen, D., Orgaz, C., Parsons, S., \& Shanks, D. R. (2020). Unconscious or underpowered? Probabilistic cuing of visual attention. Journal of Experimental Psychology: General, 149(1), 160-181. https://doi.org/10.1037/xge0000632

Vaidya, C. J., Huger, M., Howard, D. V., \& Howard, J. H. (2007). Developmental Differences in Implicit Learning of Spatial Context. Neuropsychology, 21(4), 497-506. https://doi.org/10.1037/0894-4105.21.4.497

Wang, B., Samara, I., \& Theeuwes, J. (2019). Statistical regularities bias overt attention. Attention, Perception, and Psychophysics, 81(6), 1813-1821. https://doi.org/10.3758/s13414-019-01708-5

Weintraub, S., Dikmen, S. S., Heaton, R. K., Tulsky, D. S., Zelazo, P. D., Bauer, P. J., ... Gershon, R. C. (2013). Cognition assessment using the NIH Toolbox. Neurology, 80(Issue 11, Supplement 3), S54-S64. https://doi.org/10.1212/WNL.0b013e3182872ded Won, B.-Y., \& Jiang, Y. V. (2015). Spatial working memory interferes with explicit, but not probabilistic cuing of spatial attention. Journal of Experimental Psychology: Learning, Memory, and Cognition, 41(3), 787-806. https://doi.org/10.1037/xlm0000040 
Yang, Y., \& Merrill, E. C. (2015). The impact of signal-to-noise ratio on contextual cueing in children and adults. Journal of Experimental Child Psychology, 132, 65-83. https://doi.org/10.1016/j.jecp.2014.12.005 